\title{
Multi-Layer Linkage Sealing Mechanism on Solar Cell Tool
}

\author{
Hua-xin YANG ${ }^{1,2, a}$, Yue-hui HU*1,b , Yi-Chuan $\mathrm{CHEN}^{1, \mathrm{c}}$ \\ ${ }^{1}$ Jingdezhen ceramic institute. Jingdezhen 333001 \\ ${ }^{2}$ Ideal Energy (Shanghai) Sunflower Thin Film Equipment Ltd.., Shanghai 201203,China \\ ㅁ4263028@qq.com, b8489023@163.com, lezhi2005@163.com
}

Keywords: Multi-linkage, mechanism, Dynamic Sealing, Leak Rate.

Abstract. The PECVD (Plasma Enhanced Chemical Vapor Deposition) process chamber is dynamic sealed by the Multi-layer Linkage Sealing Mechanism, which is installed in front of process boxes. The leak rate attach uniformity requirement of process gas and RF flow distribution. Meanwhile, The efficiency of aSi/uc tandem thin-film solar cell, which is produced by this tool, is higher than $12 \%$ on $1.43 \mathrm{~m}^{2}$.

\section{Introduction}

In thin film solar cell production equipment, the multi-layer process boxes are uniformly distributed into the process chamber in the vertical direction. Each of box has one entrance for substrate transferred in and out by Mechanical transmission system, for process. Process boxes need to be close by sealing parts when processing. On the other hand, the sealing parts need to be removed for the substrate transferred out from process boxes. These steps show that, sealing parts act circular motion along "open-close-open-close..." it means dynamic sealing, which drive by the mechanism called Multi-layer Linkage dynamic Sealing Mechanism. There is highly requirement for the gas pressure fluctuation and the pressure difference in PECVD process, for all the boxes [1]. It will directly affect the uniformity of the gas flow field and the electromagnetic field distribution in the chamber. Ultimately affect the process results of solar cells. Reflect to the dynamic sealing mechanism have very high requirements while the system operation of consistency and sealing property.

\section{Construction}

Fig.1 shows a multi-layer process chamber, which has six independent process boxes. The substrate is transferred in and out.

Multi-layer leakage sealing mechanism comprises with two horizontal axis, name $\mathrm{X}$ axis, two vertical axis, call $\mathrm{Y}$ axis, The $\mathrm{X}$ axis and $\mathrm{Y}$ axis are connected by hinges, shaping a rotatable parallel four-bar linkage mechanism [2]. Two $X$ axis are installed through the bearings, which are seated at both sides of chamber. Two $\mathrm{X}$ axis can rotate around their axis. One $\mathrm{X}$ axis is connected with the drive system, as a drive shaft and the driving system achieve positive and reverse rotation, so as to drive the two $\mathrm{Y}$ axis around which a circular path must be within the scope of the circumference of the translational. The other $\mathrm{X}$ axis is the driven shaft. In this case, the upper $\mathrm{X}$ axis is picked to be drive shaft, for lower $\mathrm{X}$ shaft is driven one.Fig. 2 is one layer sealing plate diagram. 


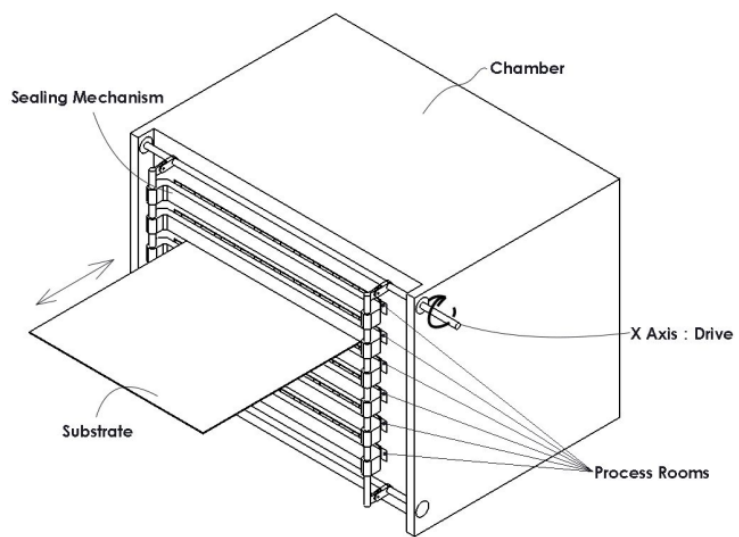

Fig.1 Chamber with Multi-layer Linkage Sealing Mechanism on open status

Mechanism contact with box surface, by the part of elastic sealing plate. The corresponding number of reactors and mutual distance, installed on the parallel four-bar linkage mechanism [2] of two $\mathrm{Y}$ axis, along $\mathrm{Y}$ axis along one circular path of translation.

Sealing plate should be "soft" material, such as aluminum, which not only avoid hardness touch, but also to make up the microscopic surface unevenness, by its deformation, for sealing property. There are numbers of spring arranger behind the sealing plate [3], whose elastic coefficient of k.

The base of the sealing plate called back plate is connected with the parallel four-bar linkage mechanism parts. The back plate should be strength material (such as stainless steel), to ensure the strength of the entire sealing plate and its shape variable. The spring is pressed and deformed, when the drive shaft is reversed to close the process boxes. The torque of the driving shaft is transformed into the sealing pressure, when the elastic force push the plate to closely attached process box.

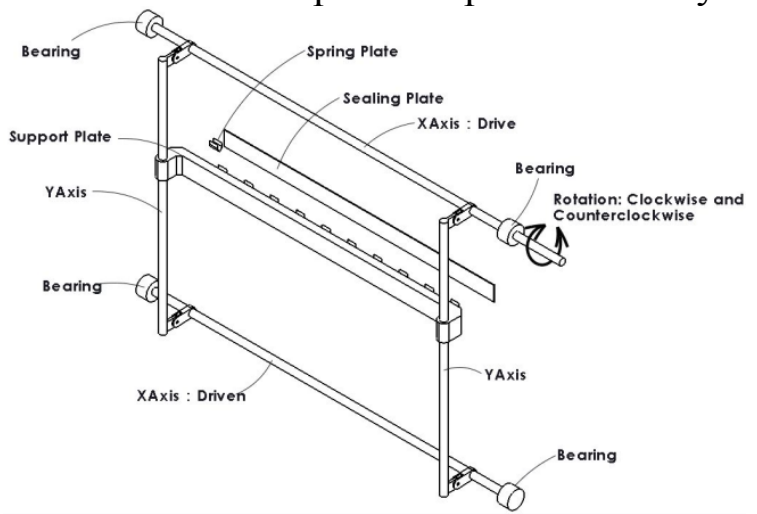

Fig.2 Multi-layer Linkage Sealing MechanismParallel Four-bar Linkage Mechanism \& Elasticity Sealing Plate

\section{Experiment}

There are some different factors affects the sealing effectiveness, through gasket sealing, such as the thickness of the gasket, width, material strength, elasticity, and pressing force, etc., The pressing force is the most particularly critical, which is related to the coefficient $\mathrm{m}$ and the pre-tightening pressure $y$. The pre-tightening pressure (or the least effective compression stress) is the compression force of the unit area effective sealing, which can make the back-up plate make elastic deformation, and is sufficient to fill in the contact surface roughness of the surface, achieve the minimum leakage rate required. The chamber pressure is constantly changing, so the final design value should be use the $\mathrm{m}$ times of the results. $\mathrm{m}$ as the coefficient of the gasket, $\mathrm{m}$ have relate with the seal of material, contact-form etc [4].The coefficient $\mathrm{m}$ and $\mathrm{y}$ are the unique values of the back-up plate itself, which can be determined by experiments.

Table 1 is a kind of commonly metal, in the case of a vessel to carry out the pre-tightening pressure test. The container is rectangular hollow, which size is close to the process box. The container is sealed by sealing element, and the pressure inside is higher than the outside, similar to the pressure difference of process box. This decline volume in a specific period of time the device internal 
pressure is the pressure drop rate, to calculate $\Delta \mathrm{P} / \Delta \mathrm{t}$, which $\Delta \mathrm{P}$ for the variation of the pressure in the interior of the container, $\Delta t$ for elapsed time, units often to $\mathrm{mbar} / \mathrm{h}$. There is relationship between the pressure drop rate and the container volume: $Q=(\Delta P / \Delta t) V$. Q as the leakage rate of the container that is an important indicator for measure of the sealing element of the container sealing effect, the unit is mbar L /s. In the experiment, the pressure difference is higher than that required by the process, and the container volume is $60 \mathrm{~L}$.

From table 1, it can be seen that the different leakage rate requires that there are different preload ratio pressure value, which provides a reference for the mechanism design.

Table 1 the Gasket Factor and Preload Pressure Ratio

\begin{tabular}{|c|c|c|c|}
\hline Material & Leak rateQ (L.mbar/s) & coefficient $\mathrm{m}$ & Pre-t press y $(\mathrm{Pa})$ \\
\hline \multirow{3}{*}{ Soft $A L$} & 3.2 & 2.00 & 4278.4 \\
\hline & 5.3 & 2.75 & 3653.6 \\
\hline & 6.1 & 3.50 & 2975.9 \\
\hline \multirow{3}{*}{ Soft Cu } & 3.2 & 2.50 & 7325.7 \\
\hline & 5.3 & 3.00 & 5896.3 \\
\hline & 6.1 & 3.25 & 5038.4 \\
\hline
\end{tabular}

\section{Calculation}

First calculate the sealing force of each layer of the sealing plate; the calculation formula is as follows.

$$
F=m \times S \times y+P \times S
$$

$\mathrm{m}$ is Gasket coefficient, $\mathrm{y}$ is Preload ratio, $\mathrm{S}$ is Effective contact area of sealing plate on the surface of opening, $\mathrm{S}$ is Entrance area, $\mathrm{P}$ is Pressure difference.

Seal pressing force of each sealing plate $\mathrm{F}$, provided by the elastic force of spring compression

$$
F=n \bar{\xi} k
$$

$\mathrm{n}$ is The number of spring for each layer of sealing plate, $\bar{\xi}$ is the average value of the compression deformation of the spring sheet. Theory of leaf spring compression amount $\xi$, determined by the position relationship, actual each spring compression amount by measuring, on the arithmetic average calculation for all the data, $\mathrm{k}$ is the elastic coefficient of the spring sheet can be measured by experiments

By shown in Figure 3, the linkage sealing mechanism carries on the stress analysis shows that the spring piece compressive deformation of the driving force which source on the driving system applied in the drive shaft torque $\mathrm{M}$, but because of each layer of sealing plate and circular translation center of different distance [5]. There is different arm, the following relationship between the torque of the whole mechanism

$$
M+G d=\sum_{i=1}^{z} n \bar{\xi} k D i
$$

$\mathrm{M}$ is the torque of rotating shaft that drive system is applied, $\mathrm{G}$ is the gravity of the linkage sealing mechanism, $d$ is horizontal distance between the center of mass and the center of rotation, Di is vertical distance between the sealing plate and the rotating center of gravity, $\mathrm{Z}$ is the number of layers of the organization. 


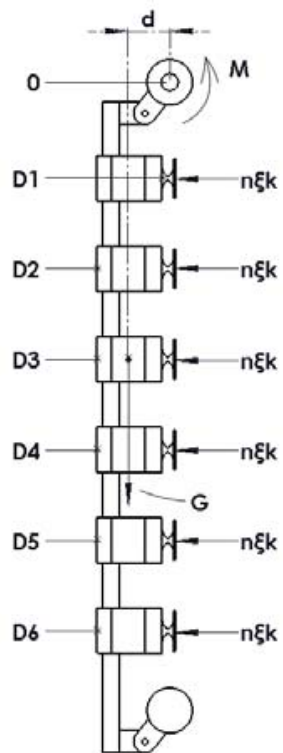

Fig.3 Force analysis of Multi-layer Linkage Sealing Mechanism M.

Synthesis (1), (2), (3) can be calculated to meet the sealing requirements of the torsion moment

\section{Examples and conclusions}

Take this design as an example, the aluminum plate is used as the sealing plate, and the effective contact area is $\mathrm{S}^{\prime}=0.04 \mathrm{~m}^{2}$, and the entrance area of each process box is $\mathrm{S}=0.05 \mathrm{~m}^{2}$.

Chamber volume $58 \mathrm{~L}$ and process cavity interior and exterior pressure difference $\mathrm{P}=5 \mathrm{mbar}$, leakage rate $\mathrm{q}$ is less than or equal to 5.0L.mbar/s, from table 1, select soft aluminum material, according to the leakage rate $\mathrm{q}$ is less than or equal to $3.2 \mathrm{~L} . \mathrm{mbar} / \mathrm{s}$, gasket coefficient $\mathrm{m}=2.00$, pre-tight pressure $y=4278.4 \mathrm{~Pa}$. According to the type of "(1)", is used to calculate the sealed pressure is $\mathrm{F}=365 \mathrm{~N}$.

Through experiments the result of measurement spring elastic coefficient $\mathrm{k}=0.08 \mathrm{~N} / \mathrm{mm}$, the theoretical amount of compression of the $\xi=30 \mathrm{~mm}$, actual measurement arithmetic average value $\bar{\xi}$ $=28.4 \mathrm{~mm}$. According to the type of "(2)", calculate each layer of sealing plate required spring number $\mathrm{n}=165$.

The vertical distance between the chamber and circle translation center: D1 $=60 \mathrm{~mm}$; D2 $=120 \mathrm{~mm}$; D3 $=180 \mathrm{~mm}$; D4=240mm; D5 $=300 \mathrm{~mm}$; D6 $=360 \mathrm{~mm}$; linkage seal mechanism has a weight of about $130 \mathrm{~kg}$. The horizontal distance of the cancroids and circle translation center $\mathrm{d}=100 \mathrm{~mm}$. According to type "(3), calculate the drive torque $\mathrm{M}=160 \mathrm{~N} . \mathrm{m}$. By the drive system provides.

The multi-rod linkage mechanism is installed into chamber to perform the sealing, and measure the chamber leakage rate when mechanism dynamic sealing, the results are as shown as table 2.The sealing effect to meet the process requirements.

Table 2 Testing result of vacuum leak-rate for multi-layer process box

\begin{tabular}{lcccccc}
\hline Chamber to P-box D & D1 & D2 & D3 & D4 & D5 & D6 \\
\hline Leak rate $($ L.mbar/s) & 3.15 & 3.26 & 3.38 & 3.42 & 3.54 & 3.63 \\
\hline
\end{tabular}

\section{Acknowledgement}

This work was financially supported by the following funds: (1) The Natural Science Foundation of China (No.61066003 and 61464005); (2) The Jiang Xi Province Key Technology R\&D Program, China (No.2010BGA01100); (3) The Natural Science Foundation of Jiangxi Province 
(No.20111BAB202005 and 20132BAB202001); (4) The Jiangxi Province Foreign Cooperation Projects, China (No.20111BDH80031 and 20132BDH80025); (5) The Leader Training Object

Project of Major Disciplines Academic and Technical of Jiangxi Province (No. 20123BCB22002), (6) The Colleges and Universities Landing Plan of Jiangxi Province (No. KJLD12085), and (7) The Education Bureau of Jiangxi Province, China (No. GJJ12494, GJJ13643 and GJJ13625).

\section{References}

[1] Weidong GU, Chao XU, Yanli LI. Study on the preparation of a-Si thin films by PECVD. Micro nano electronic Technology. Vol. 46 (2009), p. 664

[2] Cunxi XIE, Lin LI. Design and application of spatial mechanism. Mechanical Industry Press, Beijing, (2007)

[3] Xihua SHEN. Handbook of sealing materials. China Petrochemical Press, Beijing, (1991).

[4] Miaogen QIAN. Surface technology and application of materials. Machinery Industry Press, Beijing, (1990).

[5] Daxian CHENG. Handbook of mechanical design Third volume. Chemical Industry Press, Beijing, (2008). 